\title{
Thái độ đối với quảng cáo trực tuyến và ý định tiếp tục mua của người tiêu dùng: Một nghiên cứu trong ngành hàng tiêu dùng nhanh
}

\section{Attitude towards online advertising and consumer repurchase intention: A study of the fast-moving consumer goods market}

Nguyễn Đinh Yến Oanh ${ }^{1}$, Quách Lý Xuân An²*

${ }^{1}$ Trường Đại học Cần Thơ, Việt Nam

${ }^{2}$ Công ty TNHH Siam City Cement, Việt Nam

"Tác giả liên hệ, Email: an.quach@ @iamcitycement.com

THÔNG TIN

DOI: $10.46223 /$ HCMCOUJS.

econ.vi.13.2.513.2018

Ngày nhận: 10/01/2018

Ngày nhận lại: 23/01/2018

Duyệt đăng: 13/03/2018

Tù khóa:

ngành hàng tiêu dùng nhanh, nước giải khát có ga, quảng cáo trực tuyến, thái độ người tiêu dung, ý định tiếp tục mua

Keywords:

fast-moving consumer goods, carbonated drinks, online advertising, consumers' attitude, repurchase intention

\section{TÓM TÁT}

Dựa trên Thuyết hành động hợp lý TRA và mô hình Ducoffe (1996), nghiên cứu này phân tích các yếu tố tác động đến thái độ, đồng thời kiểm định ảnh hưởng của thái độ đối với quảng cáo trực tuyến đến ý định tiếp tục mua sản phẩm nước giải khát có ga. Dữ liệu được thu thập từ 557 người tiêu dùng tại Đồng bằng sông Cửu Long. Kết quả phân tích cho thấy ý định tiếp tục mua chịu ảnh hưởng bởi 3 yếu tố (1) Thái độ của người tiêu dùng đối với quảng cáo trục tuyến, (2) Giá trị cảm nhận, (3) Tính thông tin. Ngoài ra, có 4 yếu tố ảnh hưởng đến thái độ của người tiêu dùng đối với hoạt động quảng cáo trực tuyến: (1) Giá trị cảm nhận, (2) Tính thông tin, (3) Tính giải trí, (4) Danh tiếng của công ty. Kết quả nghiên cứu có ý nghĩa quan trọng về mặt học thuật và là cơ sở khoa học giúp các doanh nghiệp kinh doanh ngành hàng nước giải khát có ga giữ chân khách hàng trong thời đại số hóa, từ đó phát triển các chiến lược quảng cáo trực tuyến một cách hiệu quả.

\section{ABSTRACT}

Drawing on the Theory of Reasoned Action and Ducoffe's model (1996), this study examines the determinants of attitude and the impact of the attitude towards online advertising of carbonated drinks on consumer repurchase intention. Data were collected from 557 consumers in the Mekong Delta. The findings have pinpointed that consumer repurchase intention is influenced by Attitude, Perceived Value, and Informativeness. Besides, there are four factors affecting the attitude towards online advertising of carbonated drinks, namely: Perceived Value, Informativeness, Entertainment, and Corporate Reputation. The 
research findings offer significant contribution to theoretical literature and good references for carbonated drinks companies to retain the consumers and develop their online advertising strategies more efficiently.

\section{Giới thiệu}

Thương mại điện tử B2C của Việt Nam đã có những thay đổi quan trọng, doanh thu ước tính đạt gần 3 tỷ USD (Cục Thương mại điện tử và Công nghệ thông tin, 2014), thu hút sự quan tâm của doanh nghiệp và cộng đồng. Với bước tăng trưởng của công nghệ số, thương mại điện tử đã góp phần nâng cao hiệu quả kinh doanh cho doanh nghiệp. Việc phát triển các chiến lược tiếp thị, cung cấp nội dung thông tin đến người tiêu dùng bằng phương thức quảng cáo trực tuyến đang được doanh nghiệp sử dụng rộng rãi. Ngành hàng nước giải khát là một trong những lĩnh vực kinh doanh được ứng dụng quảng cáo trực tuyến nhiều ở thị trường Việt Nam hiện nay. Nước giải khát bao gồm nước giải khát có ga, nước giải khát không có ga, nước ép trái cây, nước đóng chai, sinh tố, cà phê và thức uống chức năng. Thị trường nước giải khát tại Việt Nam phát triển mạnh mẽ cùng với xu hướng người tiêu dùng ngày càng ưa chuộng những loại thức ăn nhanh và nước giải khát đóng chai vì tính tiện lợi và nhanh chóng. Theo Hiệp hội Bia Rượu - Nước giải khát Việt Nam, tốc độ tăng trưởng trong ngành hàng nước giải khát vào khoảng 6-7\%. Năm 2014, sản lượng nước giải khát các loại đạt 4.050 triệu lít, tăng 2,7\% so với cùng kỳ (Bộ Công Thương, 2015). Với mức tăng trưởng cao và đầy hứa hẹn, thị trường nước giải khát có ga là cơ hội đầu tư hấp dẫn của các doanh nghiệp trong và ngoài nước. Đây cũng là thách thức lớn đối với các doanh nghiệp kinh doanh trong ngành hàng này. Bên cạnh đó, xu hướng tiêu dùng cũng thay đổi so với giai đoạn trước, khi các doanh nghiệp mở rộng phát triển các sản phẩm của mình theo hướng hỗ trợ và tăng cường sức khỏe. Người tiêu dùng ngày càng chuộng các sản phẩm có nguồn gốc sạch và tự nhiên. Xu hướng này cũng được phản ánh trong việc chọn lựa nước giải khát của người tiêu dùng. Điều này lý giải vì sao các sản phẩm nước giải khát như trà xanh, trà thảo mộc ngày càng nhận được nhiều sự ưu ái của người tiêu dùng và nhanh chóng chiếm lĩnh thị trường. Đây là thách thức lớn cho ngành nước giải khát có ga. Trước tình hình hiện tại, các doanh nghiệp trong ngành nước giải khát có ga đang ra sức duy trì và lấy lại thị phần của mình, đầu tư vào các chiến dịch tiếp thị, đặc biệt là mảng quảng cáo trực tuyến. Trong đó, vấn đề không chỉ nằm ở việc thu hút khách hàng mà còn ở việc duy trì mối quan hệ để người tiêu dùng tiếp tục mua sản phẩm của doanh nghiệp. Vì vậy, làm thế nào để doanh nghiệp tận dụng hiệu quả quảng cáo trực tuyến và khách hàng tiếp tục mua sản phẩm là một trong những yếu tố then chốt quyết định sự thành công của doanh nghiệp.

Tại Việt Nam, đã có nhiều nghiên cứu tập trung vào ý định và hành vi khách hàng đối với việc mua sắm trực tuyến (T. N. Ha \& Nguyen, 2016; Ngo \& Mai, 2017; C. T. B. Nguyen \& Le, 2014; H. D. H. Nguyen, Nguyen, \& Nguyen, 2016; V. T. K. Nguyen \& Quach, 2013; T. Q. Pham \& Nguyen, 2017; Tu, 2015), thái độ đối với quảng cáo trực tuyến và ý định mua sắm (T. D. Nguyen, Tran, \& Pham, 2013; H. T. L. Pham \& Tran, 2014), hay cảm nhận bị làm phiền trong quảng cáo trực tuyến (Do, 2017). Tuy nhiên, đa số các nghiên cứu thường tập trung ở phạm vi một tỉnh, thành phố; và những mặt hàng được nghiên cứu chủ yếu là những mặt hàng được mua sắm trực tuyến nhiều như quần áo, mỹ phẩm. Trong khi đó, việc mua sắm trực tuyến 
thường không phổ biến đối với sản phẩm tiêu dùng nhanh như nước giải khát có ga. Bởi, ngành hàng tiêu dùng nhanh thường có đặc thù ở nhu cầu tiêu thụ cao nhưng cảm nhận về chi phí chuyển đổi của khách hàng thấp (Mouzas \& Naudé, 2007), nên khách hàng dễ dàng mua lại sản phẩm hoặc chuyển sang mua của nhà cung cấp khác. Theo hiểu biết tốt nhất của nhóm nghiên cứu, ảnh hưởng của thái độ đối với quảng cáo trực tuyến đến ý định của người tiêu dùng Đồng bằng sông Cửu Long về việc tiếp tục mua sản phẩm thuộc ngành hàng tiêu dùng nhanh, là chủ đề nghiên cứu vẫn còn bỏ ngỏ. Vì vậy, nghiên cứu này áp dụng mô hình Ducoffe (1996) nhằm tìm ra các yếu tố ảnh hưởng đến thái độ của người tiêu dùng Việt Nam đối với quảng cáo trực tuyến. Đồng thời, nghiên cứu này kế thừa hai yếu tố cốt lôi của mô hình TRA, TPB, TAM là thái độ - ý định và tiến hành kiểm định mối quan hệ giữa thái độ đối với quảng cáo trực tuyến nước giải khát có ga và ý định tiếp tục mua của người tiêu dùng ở Việt Nam. Việc nghiên cứu này không chỉ có đóng góp về mặt học thuật mà còn có ý nghĩa hết sức thiết thực nhằm giúp doanh nghiệp giữ chân người tiêu dùng, phát huy hiệu quả của các công cụ quảng cáo trực tuyến, từ đó góp phần tạo ra lợi thế cạnh tranh cho doanh nghiệp.

\section{Cơ sở lý thuyết và giả thuyết nghiên cứu}

\subsection{Co' sở lý thuyết}

\section{Quảng cáo trục tuyến và mô hình Ducoffe (1996)}

Một cách khái quát, quảng cáo trực tuyến (Online advertising) được hiểu là những thông điệp (Messages) có chủ ý được đặt trên trang web của một bên trung gian bao gồm cả các công cụ tìm kiếm (Search engines) và thư mục (Directories) có thể truy cập được qua Internet (L. Ha, 2008). Quảng cáo trực tuyến đã và đang phát triển dưới nhiều hình thức đa dạng nhưng websites là hình thức đầu tiên được các nhà nghiên cứu đề cập (Hwang, McMillan, \& Lee, 2003; Macias, 2003). Đề xuất xem websites như một 'kênh thương mại điện tử' đã được nhấn mạnh trong nghiên cứu của Berthon, Pitt, và Watson (1996). Đến nay, trên thế giới, đã có rất nhiều nghiên cứu về các hình thức quảng cáo trực tuyến như quảng cáo bằng banner (Banner advertising) (Breuer \& Brettel, 2012), quảng cáo bằng coupon (Coupon/loyalty advertising) (Breuer \& Brettel, 2012), quảng cáo bằng công cụ tìm kiếm (Brettel \& Spilker-Attig, 2010; Breuer \& Brettel, 2012), thư điện tử (Brettel \& Spilker-Attig, 2010), quảng cáo qua mạng xã hội (Zeng, Huang, \& Dou, 2009).

Ducoffe (1996) đã đề xuất và thử nghiệm mô hình nghiên cứu về giá trị mà quảng cáo trực tuyến mang lại và thái độ của người tiêu dùng. Kết quả nghiên cứu đã chỉ ra giá trị của quảng cáo được quyết định bởi ba yếu tố là tính giải trí, tính thông tin và tính phiền nhiễu. Hơn nữa, tính giải trí và giá trị của quảng cáo có tác động tích cực đến thái độ của người tiêu dùng đối với quảng cáo trên web. Mô hình Ducoffe (1996) đã được ứng dụng rộng rãi trong nhiều lĩnh vực như thiết kế trang web (Hausman \& Siekpe, 2009), mua sắm trực tuyến (J. U. Kim, Kim, \& Park, 2010), quảng cáo trên di động (Sinkovics, Pezderka, \& Haghirian, 2012), quảng cáo qua mạng xã hội (Logan, Bright, \& Gangadharbatla, 2012), và quảng cáo qua truyền hình (Logan et al., 2012). Tuy nhiên, mô hình Ducoffe chỉ dừng lại ở việc nghiên cứu thái độ mà chưa đi sâu khám phá tác động của các yếu tố trong mô hình đến ý định thực hiện hành vi của người tiêu dùng. Tương tự, nghiên cứu của T. D. Nguyen và cộng sự (2013) đã đề xuất mô hình chứng minh thái độ có tác động tích cực đến sự chấp nhận quảng cáo trực tuyến trên mạng xã 
hội của người tiêu dùng Việt Nam. Tuy nhiên, nghiên cứu này chưa khám phá việc thái độ đối với quảng cáo trực tuyến có tác động đến ý định mua sắm của khách hàng ở Việt Nam hay không. Nghiên cứu của Ngo và Mai (2017) phát triển mô hình do T. D. Nguyen và cộng sự (2013) đề xuất. Kết quả nghiên cứu chỉ ra rằng quảng cáo trực tuyến qua mạng xã hội có ảnh hưởng tích cực đến ý định mua sắm trực tuyến của khách hàng ở Thành phố Cần Thơ. Tuy nhiên, vai trò quan trọng của thái độ của người tiêu dùng đối với quảng cáo trực tuyến không được xem xét trong nghiên cứu vừa nêu.

\section{Lý thuyết về thái độ và ý định của ngưòi tiêu dùng}

Một trong những lý thuyết quan trọng nhất trong lĩnh vực nghiên cứu ý định và hành vi người tiêu dùng là Thuyết hành động hợp lý TRA (Theory of Reasoned Action) được Ajzen và Fishbein xây dựng (1975). Lý thuyết này cho thấy hành vi tiêu dùng (Actual behavior) được quyết định bởi ý định thực hiện hành vi đó. Ý định thực hiện hành vi chịu ảnh hưởng bởi hai yếu tố: Thái độ và ảnh hưởng xã hội đối với hành vi. Mô hình TRA đã được ứng dụng và kiểm chứng bởi rất nhiều nghiên cứu thuộc nhiều lĩnh vực khác nhau (Sheppard, Hartwick, \& Warshaw, 1988). Mô hình TRA được Ajzen (1985) mở rộng thành Thuyết hành vi dự định TPB (Theory of Planned Behavior) bằng cách bổ sung yếu tố nhận thức kiểm soát hành vi (Perceived behaviour control). Yếu tố này phản ánh việc một người nhận thức sự dễ dàng hay khó khăn khi thực hiện một hành vi (Ajzen, 1991). Mô hình TPB cho rằng, ý định thực hiện hành vi chịu tác động bởi ba yếu tố là thái độ, ảnh hưởng xã hội và nhận thức kiểm soát hành vi. Cũng dựa trên mô hình TRA, Davis, Bagozzi, và Warshaw (1989) đã phát triển mô hình chấp nhận công nghệ TAM (Technology acceptance model) để giải thích sự chấp nhận của một cá nhân đối với hệ thống thông tin. Khác với TRA, mô hình TAM không nghiên cứu tác động của yếu tố ảnh hưởng xã hội đến ý định mà chứng minh rằng, ý định không chỉ chịu tác động bởi thái độ, mà còn bởi nhận thức tính hữu dụng (Perceived usefulness). Qua đó có thể thấy, các lý thuyết nền tảng về ý định và hành vi của người tiêu dùng như TRA, TPB, TAM đều khẳng định tác động tích cực của thái độ đến ý định thực hiện hành vi. Trong lĩnh vực quảng cáo, mối quan hệ giữa thái độ và ý định từ mô hình TRA cũng đã được kiểm chứng. Chẳng hạn, nghiên cứu của Tsang, Ho, và Liang (2004) cho thấy thái độ đối với quảng cáo trên điện thoại di động và ý định mua sắm của người tiêu dùng ở Đài Loan có mối quan hệ tích cực với nhau. Tại Việt Nam, đã có nhiều nghiên cứu tập trung vào ý định và hành vi khách hàng đối với việc mua sắm trực tuyến (T. N. Ha \& Nguyen, 2016; Ngo \& Mai, 2017; C. T. B. Nguyen \& Le, 2014; H. D. H. Nguyen et al., 2016; V. T. K. Nguyen \& Quach, 2013; T. Q. Pham \& Nguyen, 2017; Tu, 2015), thái độ đối với quảng cáo trực tuyến và ý định mua sắm (T. D. Nguyen et al., 2013; H. T. L. Pham \& Tran, 2014), hay cảm nhận bị làm phiền trong quảng cáo trực tuyến (Do, 2017) (Bảng 1). Tương tự các nghiên cứu trên thế giới, kết quả nghiên cứu ở Việt Nam cũng chỉ ra mối quan hệ mật thiết giữa thái độ đối với ý định thực hiện hành vi của người tiêu dùng. 


\section{Bảng 1}

Tóm tắt một số nghiên cứu liên quan

\begin{tabular}{|c|c|}
\hline Tác giả & Mẫu \\
\hline \multicolumn{2}{|c|}{ Các nghiên cứu về ý định và hành vi mua sắn } \\
\hline C. T. B. Nguyen và & 130 người tiêu dùng ở \\
\hline V. T. K. Nguyen và & 200 cá nhân tại TP. \\
\hline Quach (2013) & $\begin{array}{l}\text { Nha Trang đã từng } \\
\text { tham gia mua bán trực } \\
\text { tuyến }\end{array}$ \\
\hline $\mathrm{Tu}(2015)$ & $\begin{array}{l}244 \text { khách hàng đã } \\
\text { từng mua sắm trực } \\
\text { tuyến }\end{array}$ \\
\hline $\begin{array}{l}\text { T. N. Ha và Nguyen } \\
\text { (2016) }\end{array}$ & $\begin{array}{l}423 \text { người có } \\
\text { kinh nghiệm sử dụng } \\
\text { Internet vào mục đích } \\
\text { mua sắm trực tuyến ở } \\
\text { Việt Nam }\end{array}$ \\
\hline $\begin{array}{l}\text { H. D. H. Nguyen và } \\
\text { cộng sự (2016) }\end{array}$ & $\begin{array}{l}500 \text { người tiêu dùng } \\
\text { có tham gia mua sắm } \\
\text { trực tuyến sinh sống tại } \\
\text { TP. HCM, Đà Nẵng và } \\
\text { Hà Nội }\end{array}$ \\
\hline
\end{tabular}

Nội dung nghiên cứu chính

Các nghiên cứu về ý định và hành vi mua sắm trực tuyến

C. T. B. Nguyen và 130 người tiêu dùng ở Ảnh hưởng của các tiêu chí tiện lợi và tiêu chí rủi ro đến hành vi mua sắm trực tuyến

V. T. K. Nguyen và Nha Trang đã từng tham gia mua bán trực tuyến từng mua sắm trực tuyến

Các yếu tố ảnh hưởng đến ý định mua sắm trực tuyến

Ý định mua sắm trực tuyến của người tiêu dùng Việt Nam

Các yếu tố tác động đến thái độ của người mua trong thị trường thương mại điện tử và mức độ ảnh hưởng của thái độ đến hành vi mua sắm trực tuyến
T. Q. Pham và Nguyen (2017)
257 người tiêu dùng ở TP. Hồ Chí Minh
Các yếu tố ảnh hưởng đến sự thôi thúc mua hàng ngẫu hứng trực tuyến

\section{Các nghiên cứu về thái độ đối với quảng cáo trực tuyến}

\section{H. T. L. Pham và Giới trẻ tại thị trường Tran (2014) Đà Nẵng \\ Các nhân tố ảnh hưởng đến thái độ của người tiêu dùng trẻ đối với quảng cáo SMS}

T. D. Nguyen và cộng sự (2013)

Ngo và Mai (2017)
280 người từng sử dụng mạng xã hội 130 người tiêu dùng ở TP. Cần Thơ
Các nhân tố tác động đến thái độ hướng đến quảng cáo qua mạng xã hội

Tác động của quảng cáo qua mạng xã hội đến ý định mua sắm 


\begin{tabular}{lll}
\hline \multicolumn{1}{c}{ Tác giả } & \multicolumn{1}{c}{ Mẫu } & \multicolumn{1}{c}{ Nội dung nghiên cứu chính } \\
\hline Do (2017) & $\begin{array}{l}\text { 160 người dùng web tại } \\
\text { TP. Hồ Chí Minh }\end{array}$ & $\begin{array}{l}\text { Nghiên cứu yếu tố ảnh hưởng và hệ quả } \\
\text { của cảm nhận bị làm phiền trong quảng } \\
\text { cáo trực tuyến }\end{array}$ \\
\hline
\end{tabular}

Nguồn: Kết quả phân tích dữ liệu của nhóm nghiên cứu

\subsection{Giả thuyết nghiên cứu}

\section{Ý định tiếp tục mua}

Ý định tiếp tục mua của người tiêu dùng đặc biệt quan trọng đối với doanh nghiệp. Các nhà quản lý luôn nỗ lực để nâng cao tỷ lệ tiếp tục mua của người tiêu dùng, bởi đây là một trong những yếu tố ảnh hưởng quan trọng đến vấn đề tài chính của doanh nghiệp trong dài hạn (Reichheld \& Sasser, 1996). Hơn nữa, những khách hàng có ý định tiếp tục mua thường có xu hướng chi trả nhiều hơn, mua số lượng lớn hơn, hoặc sẽ thu hút thêm khách hàng mới cho công ty (Ngobo, 2004), và chi phí doanh nghiệp phải dành cho quảng cáo thường thấp hơn khách hàng thông thường (Fornell et al., 2005). Theo Jones, Mothersbaugh, và Beatty (2000), trong lĩnh vực dịch vụ, khách hàng sẽ tiếp tục mua sắm nhiều hơn nếu họ cảm nhận chi phí để chuyển đổi sang nhà cung cấp khác là cao. Trong khi đó, ngành hàng tiêu dùng nhanh như ngành hàng nước giải khát có ga lại có chi phí chuyển đổi thấp (Mouzas \& Naudé, 2007), tức cảm nhận về thời gian, tiền bạc, nỗ lực mà khách hàng bỏ ra cho sản phẩm thuộc ngành hàng này không lớn (Jones et al., 2000). Nói cách khác, khách hàng có thể dễ dàng ngừng mua và chuyển sang lựa chọn khác. Vì vậy, làm thế nào để thúc đẩy ý định tiếp tục mua sắm của khách hàng là vấn đề nghiên cứu có ý nghĩa rất thiết thực trong việc giúp doanh nghiệp giữ chân khách hàng, từ đó hỗ trợ doanh nghiệp tiết kiệm được rất nhiều chi phí, mang lại lợi nhuận cao và góp phần tạo ra lợi thế cạnh tranh (Tsai \& Huang, 2007). Hiện nay, các hoạt động quảng cáo trực tuyến đã và đang khẳng định hiệu quả kết nối giữa doanh nghiệp và người tiêu dùng. Theo Thuyết hành động hợp lý TRA, thái độ có tác động tích cực đến ý định người tiêu dùng (Ajzen \& Fishbein, 1975). Do đó, trong nghiên cứu này, giả thuyết sau được đề xuất:

H1: Thái độ của nguời tiêu dùng đối với quảng cáo trục tuyến nuớc giải khát có ga có tác động tích cực đến ý định tiếp tục тиа

\section{Danh tiếng công ty, danh tiếng thương hiệu và hình ảnh thương hiệu}

Một thương hiệu được định nghĩa là tên đặc trưng hoặc biểu tượng thể hiện cho sản phẩm và dịch vụ (Aaker, 1991). Để thành công và đạt lợi nhuận cao, thương hiệu cần đạt được danh tiếng tốt (Herbig \& Milewicz, 1995). Danh tiếng thương hiệu (Brand reputation) được định nghĩa là một trong những yếu tố chính ảnh hưởng đến cảm nhận về chất lượng sản phẩm mang tên thương hiệu đó (Veloutsou \& Moutinho, 2009). Zeithaml (1988) cho rằng cảm nhận về chất lượng sản phẩm hoặc dịch vụ thường liên quan tới danh tiếng gắn liền với tên thương hiệu. Trong một số trường hợp, khách hàng thường đồng nhất một sản phẩm hoặc dịch vụ nào đó với thương hiệu (e.g., Pepsi); trong những trường hợp khác, khách hàng có thể xem một số sản phẩm là thuộc cùng một thương hiệu (e.g., IBM) (Selnes, 1993). Do đó, danh tiếng thương hiệu không nhất thiết bị giới hạn vào một sản phẩm hay dịch vụ duy nhất (Selnes, 1993). So với hình ảnh thương hiệu (Brand image), danh tiếng thương hiệu được xem là phù hợp hơn trong việc dùng để đánh giá bên ngoài đối với thương hiệu (Chernatony, 1999). Hình ảnh 
thương hiệu phản ánh nhận thức thuộc lý trí hoặc cảm xúc của khách hàng đối với những thương hiệu cụ thể (Dobni \& Zinkhan, 1990) và thường có vai trò quan trọng trong những trường hợp khó phân biệt được sản phẩm hoặc dịch vụ thông qua các tính năng hữu hình (Mudambi, Doyle, \& Wong, 1997).

Trong lĩnh vực dịch vụ, thương hiệu thường gắn liền với danh tiếng của công ty hơn là của sản phẩm hay dịch vụ đơn lẻ (Selnes, 1993). Danh tiếng công ty (Corporate reputation) được định nghĩa một cách tổng quát là kết quả của những hành động công ty đã thực hiện ( $\mathrm{N}$. Nguyen \& Leblanc, 2001), phản ánh quá trình truyền thông đến khách hàng mục tiêu thông qua chất lượng của sản phẩm và dịch vụ của nó (Yoon, Guffey, \& Kijewski, 1993). Danh tiếng được xem là tài sản vô giá của công ty, rất khó để xây dựng và rất dễ mất đi (Zhang et al., 2011). Người tiêu dùng có thể nhận thức được danh tiếng công ty thông qua hình ảnh công chúng của công ty, thương hiệu, các cam kết làm hài lòng khách hàng, sáng tạo và đổi mới trong dịch vụ khách hàng, chất lượng cung cấp sản phẩm, dịch vụ, và trách nhiệm xã hội của công ty (Qureshi et al., 2009). Như vậy, danh tiếng công ty được hiểu rộng hơn danh tiếng thương hiệu và hình ảnh thương hiệu. Danh tiếng công ty có liên quan mật thiết đến phản ứng của khách hàng đối với quảng cáo (Boateng \& Okoe, 2015). Do đó, nghiên cứu này đặt giả thuyết:

H2: Danh tiếng của công ty có tác động tích cực đến thái độ của người tiêu dùng đối với quảng cáo trục tuyến nước giải khát có ga

\section{Tính giải trí}

Tính giải trí của các hoạt động quảng cáo trực tuyến về nước giải khát có ga là mức độ cảm xúc mang lại cho người dùng khi tiếp xúc với các hoạt động quảng cáo trực tuyến $(\mathrm{T}$. $\mathrm{D}$. Nguyen et al., 2013). Nhận thức tính giải trí trong quảng cáo thể hiện qua sự thoải mái và vui vẻ thông qua trải nghiệm các kênh truyền thông (Okazaki, 2005). Theo Ducoffe (1996), tính giải trí của quảng cáo trực tuyến có thể tác động tích cực đến thái độ của người tiêu dùng. Nghiên cứu của Hoffman, Kalsbeek, và Novak (1996) cũng chỉ ra người tiêu dùng càng hài lòng và càng tương tác cao với các công cụ tiếp thị truyền thông qua mạng Internet sẽ dẫn tới thái độ tích cực và cải thiện tâm trạng của họ. Shavitt, Lowrey, và Haefner (1998) khẳng định tính giải trí cảm nhận được từ quảng cáo có ảnh hưởng mạnh đến thái độ của người tiêu dùng đối với quảng cáo đó. Điều này cũng được khẳng định trong nghiên cứu về quảng cáo trên điện thoại di động của Tsang và cộng sự (2004). Vì vậy, bài nghiên cứu đề xuất giả thuyết như sau:

H3: Tính giải trí của các hoạt động quảng cáo trục tuyến về nuớc giải khát có ga càng cao sẽ có tác động tích cực đến thái độ của người tiêu dùng

\section{Tính phiền nhiễu}

Theo Aaker và Bruzzone (1985) thì sự phiền nhiễu bao gồm sự khó chịu, phiền toái bất mãn hoặc sự kích thích tiêu cực. Tính phiền nhiễu của các hoạt động quảng cáo trực tuyến về nước giải khát có ga (Irritation) là mức độ gây khó chịu đối với người tiếp xúc với các hoạt động quảng cáo trực tuyến. Sự khó chịu này làm giảm đi đáng kể tính hiệu quả của các hoạt động quảng cáo trực tuyến và làm sai lệch đi mục tiêu ban đầu của nhà tiếp thị. Theo Ducoffe (1996), các nội dung gây xúc phạm, phản cảm sẽ ảnh hưởng tiêu cực đối với thái độ của người tiêu dùng. Dickinger, Haghirian, Murphy, và Scharl (2004) cho rằng những hoạt động quảng 
cáo trực tuyến được hiển thị nhưng người xem không mong muốn hay bằng lòng, sẽ gây nên sự phiền toái và sự không thiện cảm đến sản phẩm được tiếp thị. Do (2017) chỉ ra, việc khách hàng cảm nhận bị làm phiền sẽ tác động rõ rệt đến sự khó chịu, từ đó làm gia tăng cách nhìn tiêu cực đối với trang web. Trên cơ sở đó, bài nghiên cứu đặt giả thuyết:

H4: Tính phiền nhiễu của các hoạt động quảng cáo trục tuyến về nuớc giải khát có ga càng cao sẽ có tác động tiêu cực đến thái độ của người tiêu dùng

\section{Giá trị cảm nhận}

Khái niệm này xuất hiện vào những năm 1990 và là một trong những khái niệm được nghiên cứu nhiều đến nay (Sánchez-Fernández \& Iniesta-Bonillo, 2007). Giá trị cảm nhận được xem là giá trị mà người tiêu dùng đã cân nhắc giữa yếu tố về chất lượng sản phẩm và dịch vụ và yếu tố về chi phí tổng thể phải bỏ ra để mua sản phẩm dịch vụ đó (Fang, George, Shao, \& Wen, 2016), thể hiện thái độ và hành vi của người tiêu dùng khi tiếp tục mua một sản phẩm dịch vụ dựa vào sự đánh giá qua trải nghiệm mua trước đó (Chiu, Wang, Fang, \& Huang, 2014). Cụ thể hơn, $\mathrm{Wu}$, Chen, Chen, và Cheng (2014) cho rằng giá trị cảm nhận là sự chênh lệch giữa những lợi ích liên quan mà khách hàng đạt được (chất lượng sản phẩm, mức độ dễ dàng mua được sản phẩm) với những thứ khách hàng phải bỏ ra (công sức, tiền bạc, thời gian). Khi công ty giúp người tiêu dùng đạt được giá trị cảm nhận cao, họ kỳ vọng ý định tiếp tục mua sản phẩm, dịch vụ của người tiêu dùng sẽ cao hơn. Các nghiên cứu trước về giá trị cảm nhận đã chỉ ra khuynh hướng tiếp tục chọn mua một sản phẩm, dịch vụ khi người tiêu dùng có giá trị cảm nhận tốt (Chiu et al., 2014). Nghiên cứu này sử dụng định nghĩa giá trị cảm nhận của $W u$ và cộng sự (2014), tức là cảm nhận của người tiêu dùng về chất lượng của sản phẩm nước giải khát có ga được quảng cáo trực tuyến so với chi phí, thời gian, công sức mà người tiêu dùng phải bỏ ra để mua được sản phẩm. Nghiên cứu đặt các giả thuyết sau:

H5: Giá trị cảm nhận càng cao sẽ có tác động tích cực đến thái độ của nguời tiêu dùng

H6: Giá trị cảm nhận càng cao sẽ có tác động tích cục đến ý định tiếp tục mua của người tiêu dùng

\section{Tính thông tin}

Tính thông tin được định nghĩa là khả năng cung cấp thông tin cần thiết đến khách hàng mục tiêu (Ducoffe, 1996). Ducoffe (1996) cho rằng một hoạt động quảng cáo trực tuyến nếu cung cấp các thông tin về sản phẩm, dịch vụ đầy đủ sẽ có ảnh hưởng tích cực đến thái độ và tăng khả năng tiếp tục mua sản phẩm, dịch vụ đó. Hơn nữa, người tiêu dùng có xu hướng đánh giá nhanh chóng các thông tin họ nhận được, vì thế các thông tin cung cấp cần phải ngắn gọn, súc tích (Kaasinen, 2003). Các nghiên cứu của Ducoffe (1996), T. D. Nguyen và cộng sự (2013) đều kết luận rằng tính thông tin của quảng cáo trực tuyến có ảnh hưởng đến thái độ của người tiêu dùng. Elliot và Speck (2005) chỉ ra cảm nhận về tính thông tin có thể giúp khách hàng đưa ra quyết định tốt hơn. Trên cơ sở đó, bài nghiên cứu kiểm tra các giả thuyết sau:

H7: Tính thông tin của các hoạt động quảng cáo trục tuyến về nuớc giải khát có ga càng cao sẽ có tác động tích cực đến thái độ của người tiêu dùng 
H8: Tính thông tin của các hoạt động quảng cáo trục tuyến về nuoóc giải khát có ga càng cao sẽ có tác động tích cực đến ý định tiếp tục mua của người tiêu dùng

\section{Phương pháp nghiên cứu}

Dũ liệu được thu thập từ tháng 1/2017 đến 3/2017 thông qua khảo sát trực tuyến những người tiêu dùng đang sinh sống và làm việc ở Đồng bằng sông Cửu Long từ 6 tháng trở lên, đã từng mua nước giải khát có ga, đồng thời có tiếp xúc với các hình thức quảng cáo trực tuyến nước giải khát có ga. Phương pháp chọn mẫu thuận tiện được sử dụng trong nghiên cứu, kích thước mẫu bằng 557. Các khái niệm trong nghiên cứu được minh họa trong Bảng 2 . Để đo lường các khái niệm trong mô hình, nghiên cứu sử dụng thang đo Likert 5 mức độ từ 1 (hoàn toàn không đồng ý) đến 5 (hoàn toàn đồng ý). Kiểm định độ tin cậy của thang đo bằng hệ số Cronbach's Alpha, phân tích nhân tố khám phá EFA, phân tích nhân tố khẳng định CFA được sử dụng để đánh giá độ phù hợp của mô hình với dữ liệu thị trường. Phân tích mô hình cấu trúc tuyến tính SEM được áp dụng để kiểm định độ phù hợp của mô hình lý thuyết và các giả thuyết nghiên cứu.

\section{Bảng 2}

Các khái niệm trong nghiên cứu

\begin{tabular}{lcc}
\hline \multicolumn{1}{c}{ Khái niệm } & $\begin{array}{c}\text { Số biến } \\
\text { quan sát }\end{array}$ & Nguồn \\
\hline THÁI ĐỘ VớI QUẢNG CÁO TRỰC TUYẾN & Boateng và \\
Quảng cáo trực tuyến cho nước giải khát có ga là một ý & Okoe (2015) \\
tưởng hay & 3 \\
Tôi thích các quảng cáo nước giải khát có ga trực tuyến & \\
Nhìn chung, thái độ của tôi với quảng cáo trực tuyến về \\
nước giải khát có ga là tích cực
\end{tabular}




\begin{tabular}{|c|c|c|}
\hline Khái niệm & $\begin{array}{c}\text { Số biến } \\
\text { quan sát }\end{array}$ & Nguồn \\
\hline $\begin{array}{l}\text { TÍNH GIẢI TRÍ } \\
\text { Quảng cáo trực tuyến về nước giải khát có ga có tính giải } \\
\text { trí } \\
\text { Quảng cáo trực tuyến về nước giải khát có ga làm tôi thoải } \\
\text { mái khi xem } \\
\text { Quảng cáo trực tuyến về nước giải khát có ga thu hút tôi } \\
\text { Quảng cáo trực tuyến về nước giải khát có ga làm tôi hài } \\
\text { lòng khi xem } \\
\text { Tôi cảm thấy vui khi xem quảng cáo trực tuyến về nước } \\
\text { giải khát có ga }\end{array}$ & 5 & $\begin{array}{c}\text { Tsang và cộng } \\
\text { sự (2004) } \\
\text { Ducoffe (1995) }\end{array}$ \\
\hline $\begin{array}{l}\text { TÍNH PHIỀN NHIỄU } \\
\text { Quảng cáo trực tuyến về nước giải khát có ga làm phiền } \\
\text { người xem } \\
\text { Có quá nhiều quảng cáo trực tuyến về nước giải khát có } \\
\text { ga } \\
\text { Quảng cáo trực tuyến về nước giải khát có ga làm mất thời } \\
\text { gian của tôi } \\
\text { Nội dung trong quảng cáo trực tuyến về nước giải khát có } \\
\text { ga làm tôi khó chịu }\end{array}$ & 4 & $\begin{array}{l}\text { Tsang và cộng } \\
\text { sự (2004) } \\
\text { Ducoffe (1995) }\end{array}$ \\
\hline $\begin{array}{l}\text { GIÁ TRI CẢM NHẨN } \\
\text { Sản phẩm nước giải khát có ga được quảng cáo trực tuyến } \\
\text { có giá trị tương ứng với giá bán của nó } \\
\text { Số tiền mà tôi chi trả cho sản phẩm đó thì xứng đáng } \\
\text { Tôi không phải bỏ ra nhiều công sức để mua được nước } \\
\text { giải khát có ga được quảng cáo trực tuyến } \\
\text { Mua sản phẩm được quảng cáo trực tuyến giúp tôi tiết } \\
\text { kiệm thời gian }\end{array}$ & 4 & $\begin{array}{c}\text { Fang và cộng sự } \\
\text { (2016) } \\
\text { Wu và cộng sự } \\
\text { (2014) }\end{array}$ \\
\hline $\begin{array}{l}\text { TÍNH THÔNG TIN } \\
\text { Quảng cáo trực tuyến về nước giải khát có ga cập nhật } \\
\text { nhiều thông tin liên quan về sản phẩm } \\
\text { Quảng cáo trực tuyến về nước giải khát có ga giúp người } \\
\text { tiêu dùng thuận tiện trong việc tiếp cận thông tin sản phấm } \\
\text { Quảng cáo trực tuyến vể nước giải khát có ga cung cấp } \\
\text { thông tin về sản phẩm một cách đầy đủ } \\
\text { Quảng cáo trực tuyến về nước giải khát có ga cung cấp } \\
\text { thông tin về sản phẩm kịp thời }\end{array}$ & 4 & $\begin{array}{c}\text { Ducoffe }(1995, \\
1996)\end{array}$ \\
\hline
\end{tabular}

Nguồn: Kết quả phân tích dữ liệu của nhóm nghiên cứu

\section{Kết quả nghiên cứu}

\section{Mô tả mẫu}

Trong 557 đáp viên, có 45,4\% là nữ. Phần lớn đáp viên thuộc độ tuổi 26-35 (chiếm $45,1 \%$ ) và $18-25$ (chiếm $37,2 \%)$. Đa số đáp viên có trình độ đại học $(41,1 \%)$. Nghề nghiệp chủ yếu của đáp viên là nhân viên văn phòng $(37,5 \%)$, tự kinh doanh $(21,7 \%)$ và sinh viên $(19,6 \%)$. 
Thu nhập của đáp viên chủ yếu từ 5 đến 10 triệu/tháng (chiếm 53,3\%). Có đến 47,2\% số đáp viên dành từ 2 đến 3 giờ mỗi ngày để sử dụng mạng Internet. Hai kênh quảng cáo trực tuyến được các đáp viên tiếp xúc nhiều nhất là quảng cáo nước giải khát có ga trên trang web $(68,9 \%)$ và qua mạng xã hội $(72,2 \%)$.

\section{Kiểm định thang đo}

Kiểm định Cronbach's Alpha được sử dụng với điều kiện hệ số Cronbach's Alpha > 0,6 (Nunnally, 1978), biến nào có hệ số tương quan biến-tổng < 0,3 sẽ bị loại khỏi mô hình, và ngưng cải thiện hệ số Cronbach's Alpha nếu việc cải thiện đó không đáng kể (Hair, Black, Babin, \& Anderson, 2010). Kết quả 30 biến thuộc 7 nhân tố đều có hệ số Cronbach's Alpha > 0,6 và hệ số tương quan biến tổng của các biến $>0,3$, chứng tỏ các thang đo lường trong nghiên cứu đạt yêu cầu và không có biến nào bị loại. Do đó, mô hình gồm 29 biến quan sát ban đầu tiếp tục được sử dụng trong phân tích nhân tố khám phá.

Phân tích nhân tố khám phá EFA được tiến hành với phép quay Promax rút trích được 7 thành phần từ 29 biến quan sát. Hệ số KMO là $0,939>0,5$ nên thỏa mãn yêu cầu của phân tích nhân tố $(0,5 \leq \mathrm{KMO} \leq 1)$ (Hair et al., 2010). Kiểm định Bartlett có ý nghĩa thống kê (Sig. $=0,000<0,005)$, do đó các quan sát phù hợp cho việc phân tích nhân tố. Tổng phương sai trích giải thích được $68,43 \%$ sự biến thiên của dữ liệu, đồng thời chứng tỏ phân tích nhân tố là thích hợp (Anderson \& Gerbing, 1988). Hệ số tải nhân tố của 29 biến quan sát đều > 0,3 nên thang đo đạt yêu cầu.

Kết quả phân tích nhân tố khẳng định CFA lần thứ nhất cho thấy nhân tố tính phiền nhiễu có hiệp phương sai đáng kể với các nhân tố khác, nên nhóm biến này bị loại khỏi mô hình. Phân tích CFA được thực hiện đến lần 2 thì các chỉ số đều đạt yêu cầu, mô hình còn lại 23 biến thuộc 6 nhân tố. Kết quả kiểm định Chi-bình phương của mô hình tới hạn có giá trị Pvalue $=0,000<0,05$, các chỉ tiêu mô hình có Chi-square/df $=2,854<3$ (Carmines \& McIver, 1981), RMSEA=0,058 < 0,08 (Steiger, 1990), GFI=0,905 > 0,9, TLI=0,947 > 0,9, CFI=0,955 $>0,9$ (Bentler \& Bonett, 1980) hay các chỉ tiêu đều đạt yêu cầu nên mô hình phù hợp với dữ liệu thị trường.

Các trọng số chưa chuẩn hóa đều có ý nghĩa thống kê (p-value < 0,05), các trọng số đã chuẩn hóa đều > 0,5 (Hair et al., 2010), phương sai trích của các nhân tố đều > 0,5 (Fornell \& Larcker, 1981) nên thang đo đạt giá trị hội tụ.

Hệ số tương quan các biến đều < 1 nên các biến không có tương quan với nhau, mô hình đạt tính đơn nguyên. Hệ số tương quan của từng cặp biến < 1 ở độ tin cậy $95 \%$ và p-value đều < 0,05 nên các khái niệm đạt tính phân biệt.

Độ tin cậy tổng hợp đều > 0,7 (trừ nhân tố Tính thông tin) (Nunnally \& Bernstein, 1994) và Cronbach's Alpha của các nhân tố cũng đều $>0,6$ (Nunnally, 1978) nên các thang đo đều đạt độ tin cậy tốt (Bảng 3). 


\section{Bảng 3}

Kết quả kiểm định sự phù hợp của mô hình

\begin{tabular}{lcccc}
\hline \multicolumn{1}{c}{ Khái niệm } & $\begin{array}{c}\text { Số biến } \\
\text { quan } \\
\text { sát }\end{array}$ & $\begin{array}{c}\text { Độ tin } \\
\text { cậy } \\
\text { tổng hợp }\end{array}$ & $\begin{array}{c}\text { Phương } \\
\text { sai trích }\end{array}$ & $\begin{array}{c}\text { Cronbach's } \\
\text { Alpha }\end{array}$ \\
\hline Thái độ với quảng cáo trực tuyến & 3 & 0,905 & 0,761 & 0,904 \\
Ý định tiếp tục mua & 3 & 0,891 & 0,732 & 0,891 \\
Danh tiếng & 5 & 0,920 & 0,698 & 0,919 \\
Tính giải trí & 5 & 0,834 & 0,506 & 0,833 \\
Giá trị cảm nhận & 4 & 0,862 & 0,610 & 0,873 \\
Tính thông tin & 4 & 0,644 & 0,578 & 0,859 \\
\hline
\end{tabular}

Nguồn: Kết quả xử lý dữ liệu điều tra

\section{Kiểm định mô hình}

Kết quả mô hình cấu trúc tuyến tính thể hiện mức độ phù hợp của mô hình với dữ liệu thị trường với 213 bậc tự do; Chi-square/df=2,513 < 3 (Carmines \& McIver, 1981), RMSEA=0,052 < 0,08 (Steiger, 1990), GFI=0,921 > 0,9; TLI=0,957 > 0,9; CFI=0,964 > 0,9 (Bentler \& Bonett, 1980). Như vậy, các giả thuyết trong mô hình (H1, H3, H4, H5, H6, H7, $\mathrm{H} 8$ ) đều được ủng hộ, trừ giả thuyết H2. Các trọng số chưa chuẩn hóa và chuẩn hóa đều dương, cho thấy các mối quan hệ này cùng chiều (Bảng 4).

\section{Bảng 4}

Kết quả kiểm định giả thuyết

\begin{tabular}{clllllc}
\hline $\begin{array}{c}\text { Giả } \\
\text { thuyết }\end{array}$ & \multicolumn{2}{c}{ Mối quan hệ } & $\boldsymbol{\beta}$ & $\mathbf{p}$ & $\begin{array}{c}\text { Kiểm định } \\
\text { giả thuyết }\end{array}$ \\
\hline H1 & Thái độ & $\rightarrow$ & Ý định tiếp tục mua & 0,449 & 0,000 & Unng hộ \\
H2 & Danh tiếng & $\rightarrow$ & Thái độ & 0,133 & 0,010 & Unng hộ \\
H3 & Tính giải trí & $\rightarrow$ & Thái độ & 0,258 & 0,000 & Ủng hộ \\
H4 & Tính phiền nhiễu & $\rightarrow$ & Thái độ & & & Không ủng hộ \\
H5 & Giá trị cảm nhận & $\rightarrow$ & Thái độ & 0,321 & 0,000 & Ủng hộ \\
H6 & Giá trị cảm nhận & $\rightarrow$ & Ý định tiếp tục mua & 0,315 & 0,000 & Unng hộ \\
H7 & Tính thông tin & $\rightarrow$ & Thái độ & 0,262 & 0,000 & Unng hộ \\
H8 & Tính thông tin & $\rightarrow$ & Ý định tiếp tục mua & 0,094 & 0,092 & Unng hộ \\
\hline
\end{tabular}

Nguồn: Kết quả xử lý dữ liệu điều tra

\section{Thảo luận kết quả}

Ý định tiếp tục mua nước giải khát có ga chịu ảnh hưởng bởi ba nhân tố: Giá trị cảm nhận, tính thông tin và thái độ. Thái độ của người tiêu dùng Đồng bằng sông Cửu Long đối với quảng cáo trực tuyến chịu ảnh hưởng của bốn nhân tố: Danh tiếng, tính giải trí, giá trị cảm nhận và tính thông tin. Ngoài ra, nhân tố tính phiền nhiễu không có tác động đáng kể đến thái độ người tiêu dùng.

Giá trị cảm nhận: Là nhân tố có tác động mạnh nhất tới thái độ của người tiêu dung $(\beta=0,321, p=0,000)$. Bên cạnh đó, nó cũng tác động tới ý định tiếp tục mua nước giải khát có 
ga của người tiêu dùng $(\beta=0,315, \mathrm{p}=0,000)$. Kết quả này phù hợp với các nghiên cứu trong lĩnh vực thương mại điện tử (Fang et al., 2016). Nhân tố giá trị cảm nhận được công nhận là một trong những nhân tố quan trọng trong việc xác định các yếu tố ảnh hưởng đến ý định tiếp tục mua một số ngành hàng tiêu dùng nhất định (Luk, Sharma, \& Chen, 2013).

Tính thông tin: Nhân tố tác động mạnh thứ hai đến thái độ của người tiêu dùng $(\beta=0,262$, $\mathrm{p}=0,000$ ). Điều này phù hợp với các nghiên cứu về thái độ của người tiêu dùng đối với các quảng cáo trực tuyến như nghiên cứu về thái độ hướng đến quảng cáo của Ducoffe (1996), T. D. Nguyen và cộng sự (2013), Ngo và Mai (2017). Do đó, khi các hình thức quảng cáo trực tuyến càng tăng tính thông tin có ích cho người tiêu dùng thì thái độ của họ sẽ tích cực hơn. Thông tin truyền tải cần ngắn gọn, súc tích và mang nhiều thông tin tích cực cho người tiêu dùng. Ngoài ra, tính thông tin cũng có ảnh hưởng đến ý định của người tiêu dung ở mức ý nghĩa $10 \%$; tuy nhiên, tác động này không đáng kể $(\beta=0,094, \mathrm{p}=0,092)$.

Tính giải trí: Nhân tố tác động mạnh thứ ba đến thái độ người tiêu dùng khi tiếp xúc với các hoạt động quảng cáo trực tuyến về nước giải khát có ga $(\beta=0,258 ; \mathrm{p}=0,000)$. Điều này phù hợp với các nghiên cứu về thái độ của người tiêu dùng đối với quảng cáo trực tuyến của Ko và Roberts (2005), thái độ hướng đến quảng cáo của Ducoffe (1996), T. D. Nguyen và cộng sự (2013), Ngo và Mai (2017). Kết quả gợi ý rằng khi một hoạt động quảng cáo trực tuyến làm gia tăng mức độ thích thú và lôi cuốn thì người tiêu dùng sẽ có thái độ tích cực hơn.

Danh tiếng của công ty: Ảnh hưởng tích cực đến thái độ của người tiêu dùng $(\beta=0,133$ và $\mathrm{p}=0,010$ ), qua đó gián tiếp ảnh hưởng đến ý định tiếp tục mua. Kết quả phù hợp với các nghiên cứu trước đây trong nhóm ngành $\mathrm{B} 2 \mathrm{C}$ (nhóm doanh nghiệp - khách hàng) có ứng dụng quảng cáo trực tuyến trong thương mại điện tử (M. K. Kim, Park, \& Jeong, 2004). Kết quả gợi ý rằng khi công ty có danh tiếng tốt thì các hoạt động quảng cáo của họ sẽ nhận được thái độ tích cực từ người tiêu dùng.

Thái độ đối với hoạt động quảng cáo trục tuyến: Nhân tố ảnh hưởng mạnh nhất tới ý định tiếp tục mua nước giải khát có ga $(\beta=0,449 ; \mathrm{p}=0,000)$. Kết quả nghiên cứu này một lần nữa khẳng định mối quan hệ giữa thái độ và ý định mua lặp lại đối với ngành hàng nước giải khát có ga tại Việt Nam, phù hợp với lý thuyết hành động hợp lý TRA của Ajzen và Fishben (1975). Khi người tiêu dùng có thái độ tích cực về hoạt động quảng cáo trực tuyến của một công ty nào đó, ý định tiếp tục mua sản phẩm nước giải khát của công ty đó sẽ tăng lên.

Tính phiền nhiễu: Trái với đa số các nghiên cứu trước (Ducoffe, 1995; Ducoffe, 1996; Ngo \& Mai, 2017; Tsang et al., 2004), tính phiền nhiễu của các hoạt động quảng cáo trực tuyến về nước giải khát có ga không có tác động đáng kể đến thái độ của người tiêu dùng trong bối cảnh của nghiên cứu này. Tuy nhiên, kết quả này tương đồng với các nghiên cứu gần đây về mối quan hệ giữa tính phiền nhiễu và thái độ. Nghiên cứu mới đây của Do (2017) cho thấy, không tồn tại tác động rõ ràng của cảm nhận bị làm phiền đến thái độ tiêu cực đối với trang web của người dùng web tại Thành phố Hồ Chí Minh. Tương tự, Aydin và Karamehmet (2017) cũng kết luận rằng tính phiền nhiễu không ảnh hưởng đến thái độ của người tiêu dùng Thổ Nhĩ Kỳ đối với quảng cáo trên ứng dụng di động. Điều này có thể giải thích bởi khách hàng có thể chọn lựa giữa ứng dụng trả phí (không quảng cáo) và ứng dụng miễn phí (có quảng cáo). Do đó, việc khách hàng chọn lựa ứng dụng miễn phí thường đồng nghĩa với việc khách hàng chấp 
nhận ứng dụng đó chứa đựng quảng cáo. Chowdhury, Parvin, Weitenberner, và Becker (2006) chỉ ra rằng, nếu các quảng cáo được hiển thị với nội dung phù hợp, khách hàng có thể không cảm thấy phiền phức và thậm chí có khả năng xem xét các quảng cáo mà họ cho là liên quan. Do (2017) cũng đề xuất các quảng cáo trực tuyến nên hiển thị với kích thước và vị trí phù hợp để tránh tạo cảm giác người xem bị tấn công dồn dập.

\section{Kết luận}

Kết quả nghiên cứu 557 người tiêu dung ở Đồng bằng sông Cửu Long cho thấy ảnh hưởng tích cực thái độ của người tiêu dung đối với các hoạt động quảng cáo trực tuyến nước giải khát có ga đến ý định tiếp tục mua của khách hàng. Thái độ đối với quảng cáo trực tuyến và ý định, hành vi của người tiêu dùng đã được nghiên cứu nhiều ở cả trong và ngoài nước. Tuy nhiên, việc nghiên cứu ý định tái mua sắm của khách hàng trong mối quan hệ với quảng cáo trực tuyến nước giải khát có ga ở Việt Nam là chủ đề nghiên cứu còn mới. Về mặt lý thuyết, đây là nghiên cứu đầu tiên sử dụng mô hình Ducoffe (1996) và Thuyết hành động hợp lý TRA để kiểm định mối quan hệ giữa thái độ người tiêu dùng đối với quảng cáo trực tuyến với ý định tiếp tục mua nước giải khát có ga của người tiêu dùng Đồng bằng sông Cửu Long. Kết quả nghiên cứu chỉ ra: Thứ nhất, thái độ của người tiêu dùng Đồng bằng sông Cửu Long đối với quảng cáo trực tuyến chịu ảnh hưởng của bốn nhân tố là danh tiếng công ty, tính giải trí, giá trị cảm nhận và tính thông tin. Nhân tố tính phiền nhiê̂u không tác động đáng kể đến thái độ của người tiêu dùng trong bối cảnh của nghiên cứu này. Thứ hai, nếu nghiên cứu của $T$. $D$. Nguyen và cộng sự (2013) mới khám phá các nhân tố tác động đến thái độ hướng đến quảng cáo của người tiêu dùng, thì nghiên cứu này đã tiến tới khẳng định tồn tại mối quan hệ tích cực giữa thái độ đối với quảng cáo trực tuyến đến ý định tiếp tục mua của người tiêu dùng ở Đồng bằng sông Cửu Long. Kết quả này cũng khẳng định mối quan hệ giữa thái độ - ý định của Thuyết hành động hợp lý TRA khi áp dụng vào trường hợp nghiên cứu ngành hàng tiêu dùng nhanh. Thứ ba, nếu mô hình Ducoffe (1996) chỉ tìm ra mối quan hệ gián tiếp giữa tính thông tin đối với thái độ, thì nghiên cứu này đã khám phá được mối quan hệ tác động trực tiếp và cùng chiều giữa tính thông tin đến thái độ người tiêu dùng. Thứ tu;, nghiên cứu này cũng chứng minh được ý định tiếp tục mua của khách hàng chịu ảnh hưởng bởi các yếu tố thái độ, giá trị cảm nhận, tính thông tin. Các nhà nghiên cứu có thể tham khảo mô hình trong nghiên cứu này để phát triển các hướng nghiên cứu tiếp theo về hành vi khách hàng trong bối cảnh quảng cáo trực tuyến đang phát triển mạnh ở Việt Nam. Với sự cạnh tranh gay gắt trong ngành hàng nước giải khát ở Việt Nam hiện nay, kết quả nghiên cứu không chỉ có ý nghĩa về mặt lý thuyết mà còn rất thiết thực trong việc hỗ trợ doanh nghiệp nắm bắt thái độ và ý định của người tiêu dùng, phát huy hiệu quả của quảng cáo trực tuyến; từ đó, góp phần nâng cao khả năng cạnh tranh.

\section{Hàm ý quản trị}

Kết quả nghiên cứu có thể thúc đẩy sự quan tâm của doanh nghiệp và các chuyên gia tiếp thị đến thái độ của khách hàng đối với quảng cáo trực tuyến sản phẩm thuộc ngành hàng tiêu dùng nhanh, nhận biết các yếu tố hình thành nên thái độ và ý định tiếp tục mua của khách hàng, từ đó xây dựng các giải pháp để nâng cao hiệu quả của các chiến dịch quảng cáo trực tuyến đến người tiêu dùng. Trên cơ sở kết quả của bài nghiên cứu, tác giả để xuất một số hàm 
ý quản trị nhằm nâng cao hiệu quả của quảng cáo trực tuyến trong việc đẩy mạnh ý định tiếp tục mua của người tiêu dùng như sau:

Thứ nhất, các thông tin được truyền tải trong các quảng cáo trực tuyến cần đầy đủ, súc tích và cần hỗ trợ tích cực cho quyết định tiếp tục chọn mua nước giải khát. Bên cạnh đó, người tiêu dùng ngày càng khá quen thuộc với các thông tin về nước giải khát, chính vì thế các thông điệp truyền tải cần mang tính mới lạ, độc đáo. Thứ hai, tăng cường yếu tố giải trí của quảng cáo trực tuyến. Cần tập trung xây dựng những nội dung mới, hấp dẫn, kích thích trí tò mò của người xem. Bên cạnh đó có thể kết hợp sử dụng hình ảnh người nổi tiếng để đẩy mạnh thêm yếu tố giải trí trên các hoạt động quảng cáo trực tuyến. Chẳng hạn, trong khi những clip hướng dẫn an toàn bay của các hãng hàng không thường không mấy hấp dẫn, thì quảng cáo của hãng hàng không Air New Zealand năm 2014 đã trở thành một trong những clip quảng cáo hàng không được yêu thích nhất, thu hút hơn 18 triệu lượt xem trên Youtube tính đến cuối năm 2017. Cụ thể, đoạn video với tên gọi The Most Epic Safety Video Ever Made không chỉ chứa đựng tính thông tin cao về an toàn bay, mà còn có tính giải trí cao với sự xuất hiện của các diễn viên trong bộ phim nổi tiếng The Hobbit, cùng những cảnh đẹp hùng vĩ ở New Zealand.

Thứ $\boldsymbol{b a}$, cần nâng cao giá trị nhận thức về sản phẩm nước giải khát có ga sao cho người tiêu dùng cảm nhận được số tiền mua sản phẩm phải thật sự tương xứng. Để làm được điều đó, các doanh nghiệp cần tập trung truyền thông về giá trị của sản phẩm và những lợi ích mà người tiêu dùng có thể đạt được khi mua sắm sản phẩm, có thể bao gồm: các giá trị lợi ích về mặt sức khỏe như hỗ trợ tiêu hóa, cung cấp năng lượng, hay thể hiện phong cách sống hiện đại. Đồng thời, cần chú trọng đầu tư vào bao bì sản phẩm với thiết kế hiện đại, độc đáo thu hút được sự chú ý của người tiêu dùng. Chẳng hạn, chiến dịch 'Shake a coke' của Coca-Cola đã thành công rực rỡ nhờ cho phép người mua tự tạo ra một lon coca có in tên riêng cho mình hoặc cho người khác. Qua đó, khách hàng không chỉ nhận được sản phẩm khi mua mà còn được cá nhân hóa sản phẩm và được chia sẻ giá trị đó với các khách hàng khác.

Thứ tur, chú trọng việc củng cố danh tiếng của công ty. Doanh nghiệp cần đẩy mạnh thêm yếu tố nhận diện thương hiệu trong các hoạt động quảng cáo trực tuyến. Các logo, hình ảnh, khẩu hiệu cần thống nhất thành một bộ nhận diện để người dùng dễ dàng nhận biết ngay khi tiếp xúc với các quảng cáo trực tuyến. Một trong những ví dụ điển hình là Ngân hàng $\mathrm{ANZ}$, doanh nghiệp này đã tiến hành nghiên cứu để thiết kế lại logo và bộ nhận diện thương hiệu, nhằm tăng sự nhận biết và tính trải nghiệm của khách hàng ở 3 thị trường chính là Úc, New Zealand và châu Á Thái Bình Dương. Doanh nghiệp cũng cần lưu ý việc sử dụng bộ nhận diện thương hiệu cần phải tiết chế một cách khéo léo, tinh tế, tránh gây phản cảm hoặc tạo hiệu ứng ngược đối với thái độ của người tiêu dùng.

\section{Hạn chế và hướng nghiên cứu tiếp theo}

Bên cạnh những kết quả đạt được, nghiên cứu này cũng có những hạn chế nhất định. Mô hình nghiên cứu mới chỉ ra được một phần các nhân tố ảnh hưởng đến ý định tiếp tục mua sản phẩm mà chưa đề cập đến hành vi mua thật sự và chưa tập trung vào một hình thức, quảng cáo trực tuyến cụ thể. Để nâng cao khả năng tổng quát hóa kết quả nghiên cứu, các nghiên cứu tiếp theo nên mở rộng phạm vi thu thập số liệu, chọn mẫu theo xác suất, đồng thời nghiên cứu mối quan hệ giữa ý định và hành vi mua thật sự của người tiêu dùng đối với từng hình thức 
quảng cáo trực tuyến cụ thể. Ngoài ra, nghiên cứu này chỉ sử dụng phương pháp định lượng để kiểm định giả thuyết. Phương pháp này thường tập trung vào việc kiểm định dựa vào quy trình suy diễn (T. D. Nguyen, 2011), nhằm lượng hóa và đo lường các mối quan hệ giữa các biến. Các nghiên cứu tiếp theo có thể kiểm định lại kết quả nghiên cứu này theo cách tiếp cận dựa vào quy trình quy nạp (sử dụng phương pháp định tính) hoặc kết hợp phương pháp định lượng và định tính (hỗn hợp).

\section{Tài liệu tham khảo}

Aaker, D. A. (1991). Managing brand equity: Capitalizing on the value of a brand name. New York, NY: Free Press.

Aaker, D. A., \& Bruzzone, D. E. (1985). Causes of irritation in advertising. Journal of Marketing, 49(2), 47-57.

Ajzen, I. (1985) From intentions to actions: A theory of planned behavior. In J. Kuhl \& J. Beckmann (Eds.), Action control : From cognition to behavior (pp. 11-39). doi:0.1007/978-3-642-69746-3_2

Ajzen, I. (1991). The theory of planned behavior. Organizational Behavior and Human Decision Processes, 50(2), 179-211.

Ajzen, I., \& Fishbein, M. (1975). Belief, attitude, intention and behavior: An introduction to theory and research. Reading, MA: Addison-Wesley Publishing Company.

Anderson, J. C., \& Gerbing, D. W. (1988). Structural equation modeling in practice: A review and recommended two-step approach. Psychological Bulletin, 10(3), 411-423.

Aydin, G., \& Karamehmet, B. (2017). A comparative study on attitudes towards SMS advertising and mobile application advertising. International Journal of Mobile Communications, 15(5), 514-536.

Bentler, P. M., \& Bonett, D. G. (1980). Significance tests and goodness of fit in the analysis of covariance structures. Psychological Bulletin, 88(3), 588-606.

Berthon, P., Pitt, L. F., \& Watson, R. T. (1996). The World Wide Web as an advertising medium. Journal of Advertising Research, 36(1), 43-54.

Bộ Công Thương. (2015). Hiệp hội Bia - Rươu - Nước giải khát Việt Nam hoàn thành vurợt mức các chi tiêu kế hoạch 2014 [The Vietnam Beer - Wine - Beverage Association has exceeded the 2014 target targets]. Retrieved from October 12, 2017, from http://www.moit.gov.vn/vn/tin-tuc/4653/hiep-hoi--bia--ruou-- nuoc-giai-khat-viet-namhoan-thanh-vuot-muc-cac-chi-tieu-ke-hoach-2014.aspx

Boateng, H., \& Okoe, A. F. (2015). Consumers attitude towards social media advertising and their behavioural response: The moderating role of corporate reputation. Journal of Research in Interactive Marketing, 9(4), 299-312.

Brettel, M., \& Spilker-Attig, A. (2010). Online advertising effectiveness: A cross-cultural comparison. Journal of Research in Interactive Marketing, 4(3), 176-196. 
Breuer, R., \& Brettel, M. (2012). Short-and long-term effects of online advertising: Differences between new and existing customers. Journal of Interactive Marketing, 26(3), 155-166.

Carmines, E. G., \& McIver, J. P. (1981). Analyzing Models with unobserved variables: Analysis of covariance structures. In G. W. Bohrnstedt \& E. F. Borgatta (Eds.), Social measurement: Current issues (pp. 65-115). Beverly Hills, CA: Sage Publications.

Chernatony, L. D. (1999). Brand management through narrowing the gap between brand identity and brand reputation. Journal of Marketing Management, 15(1/3), 157-179.

Chiu, C., Wang, E., Fang, Y., \& Huang, H. (2014). Understanding customers repeat purchase intentions in B2C e-commerce: The roles of utilitarian value, hedonic value and perceived risk. Information Systems Journal, 24, 85-114.

Chowdhury, H. K., Parvin, N., Weitenberner, C., \& Becker, M. (2006). Consumer attitude toward mobile advertising in an emerging market: An empirical study. International Journal of Mobile Marketing, 1(1), 30-42.

Cục Thương mại điện tử và Công nghệ thông tin. (2014). Báo cáo Thuoong mại điện tử Việt Nam 2014 [Vietnam E-Commerce report 2014]. Retrieved October 13, 2017, from http://www.moit.gov.vn/documents/20182/1577125/1508897822937-709f2858-88e2427c-9bbc-48d2cb974902.pdf?download=true

Davis, F. D., Bagozzi, R. P., \& Warshaw, P. R. (1989). User acceptance of computer technology: A comparison of two theoretical models. Management Science, 35(8), 9821003.

Dickinger, A., Haghirian, P., Murphy, J., \& Scharl, A. (2004). An investigation and conceptual model of SMS marketing. In System sciences, 2004. Proceedings of the 37th Annual Hawaii International Conference on (pp. 1-10). New York, NY: IEEE.

Do, H. T. L. (2017). Nghiên cứu yếu tố ảnh hưởng và hệ quả của cảm nhận bị làm phiền trong quảng cáo trực tuyến [Study the influencing factors and consequences of the perceived disturbance in online advertising]. Tạp chí Khoa hoc Đại học Mở Thành phố Hồ Chí Minh, 56(5), 116-130.

Dobni, D., \& Zinkhan, G. M. (1990). In search of brand image: A foundation analysis. ACR North American Advances, 17, 110-119.

Ducoffe, R. H. (1995). How consumers assess the value of advertising. Journal of Current Issues \& Research in Advertising, 17(1), 1-18.

Ducoffe, R. H. (1996). Advertising value and advertising on the web. Journal of Advertising Research, 36(5), 21-21.

Elliot, M. T., \& Speck, P. S. (2005). Factors that affect attitude toward a retail website. Journal of Marketing Theory and Practice, 13(1), 40-51.

Fang, J., George, B., Shao, Y., \& Wen, C. (2016). Affective and cognitive factors influencing repeat buying in e-commerce. Electronic Commerce Research and Applications, 19, 4455.

Fornell, C., \& Larcker, D.F. (1981). Evaluating structural equation models with unobservable variables and measurement error. Journal of Marketing Research, 18, 39-50. 
Fornell, C., VanAmburg, D., Morgeson, F. V., Anderson, E. W., Bryant, B. E., \& Johnson, M. D. (2005). The American customer satisfaction index at ten years. Ann Arbor, MI: Stephen M. Ross School of Business.

Ha, L. (2008). Online advertising research in advertising journals: A review. Journal of Current Issues \& Research in Advertising, 30(1), 31-48.

Ha, T. N., \& Nguyen, D. T. (2016). Các yếu tố ảnh hưởng đến ý định mua sắm trực tuyến của người tiêu dùng Việt Nam: Nghiên cứu mở rộng thuyết hành vi có hoạch định [Factors affecting online shopping intentions of Vietnamese consumers: Extensive study of planned behavioral theory]. Tạp chí Khoa hoc ĐHQGHN: Kinh tế và Kinh doanh, 32(4), $21-28$

Hair, J., Black, W., Babin, B., \& Anderson, R. (2010). Multivariate data analysis (7th ed.). Upper Saddle River, NJ: Prentice-Hall, Inc.

Hausman, A. V., \& Siekpe, J. S. (2009). The effect of web interface features on consumer online purchase intentions. Journal of Business Research, 62(1), 5-13.

Herbig, P., \& Milewicz, J. (1995). The relationship of reputation and credibility to brand success. Journal of Consumer Marketing, 12(4), 5-10.

Hoffman, D. L., Kalsbeek, W. D., \& Novak, T. P. (1996). Internet and Web use in the US. Communications of the ACM, 39(12), 36-46.

Hwang, J. S., McMillan, S. J., \& Lee, G. (2003). Corporate web sites as advertising: An analysis of function, audience, and message strategy. Journal of Interactive Advertising, 3(2), 1023.

Jones, M. A., Mothersbaugh, D. L., \& Beatty, S. E. (2000). Switching barriers and repurchase intentions in services. Journal of Retailing, 76(2), 259-274.

Kaasinen, E. (2003). User needs for location-aware mobile services. Personal and Ubiquitous Computing, 7(1), 70-79.

Kim, J. U., Kim, W. J., \& Park, S. C. (2010). Consumer perceptions on web advertisements and motivation factors to purchase in the online shopping. Computers in Human Behavior, 26(5), 1208-1222.

Kim, M. K., Park, M. C., \& Jeong, D. H. (2004). The effects of customer satisfaction and switching barrier on customer loyalty in Korean mobile telecommunication services. Telecommunications Policy, 28(2), 145-159.

Ko, H., Cho, C. H., \& Roberts, M. S. (2005). Internet uses and gratifications: A structural equation model of interactive advertising. Journal of Advertising, 34(2), 57-70.

Logan, K., Bright, L. F., \& Gangadharbatla, H. (2012). Facebook versus television: Advertising value perceptions among females. Journal of Research in Interactive Marketing, 6(3), 164-179.

Luk, S. T. K., Sharma, P., \& Chen, I. S. N. (2013). Shopping motivation as a moderator in the retail service evaluation. Journal of Services Marketing, 27(1), 40-48. 
Macias, W. (2003). A beginning look at the effects of interactivity, product involvement and web experience on comprehension: Brand web sites as interactive advertising. Journal of Current Issues \& Research in Advertising, 25(2), 31-44.

Mouzas, S., \& Naudé, P. (2007). Network mobilizer. Journal of Business \& Industrial Marketing, 22(1), 62-71.

Mudambi, S. M., Doyle, P., \& Wong, V. (1997). An exploration of branding in industrial markets. Industrial Marketing Management, 26(5), 433-446.

Ngo, T. M., \& Mai, T. V. N. (2017). Phân tích tác động của quảng cáo qua mạng xã hội đến ý định mua sắm của người tiêu dùng tại thành phố Cần Thơ [Analyzing the impact of social media advertising on consumers' shopping intentions in Can Tho City]. Tạp chi Khoa hoc Truò̀ng Đại học Cần Tho, 48d, 66-76.

Ngobo, V. P. (2004). Drivers of customers cross-buying intentions. European Journal of Marketing, 38(9/10), 1129-1157.

Nguyen, C. T. B., \& Le, D. X. N. (2014). Phân tích các nhân tố ảnh hưởng đến hành vi mua sắm trực tuyến của người tiêu dùng Thành phố Cần Thơ [Analysis of factors affecting online shopping behavior of Can Tho City consumers]. Tạp chí Khoa hoc Truờng Đại hoc Cần Tho; 30, 8-14.

Nguyen, H. D. H., Nguyen, M. T. B., \& Nguyen, T. N. B. (2016). Nghiên cứu các yếu tố ảnh hưởng đến thái độ của người mua trong thị trường thương mại điện tử [Study the factors that influence buyers' attitudes in the e-commerce market]. Tạp chí Phát triển Khoa học và Công nghẹ, 19, 68-80.

Nguyen, N., \& Leblanc, G. (2001). Corporate image and corporate reputation in customer retention decisions in services. Journal of Retailing and Consumer Services, 8(4), 227236.

Nguyen, T. D. (2011). Phwơng pháp nghiên cúu khoa hoc trong kinh doanh [Scientific research method in business]. Hanoi, Vietnam: Nhà xuất bản Lao động - Xã hội.

Nguyen, T. D., Tran, N. D., \& Pham, C. M. (2013). Đề xuất mô hình chấp nhận quảng cáo trực tuyến trên mạng xã hội ởViệt Nam [Proposing an acceptable model for online advertising on social networks in Vietnam]. Tạp chí Phát triển Khoa học và Công nghệ, 16, 5-19.

Nguyen, V. T. K., \& Quach, N. T. K. (2013). Các nhân tố ảnh hưởng đến thái độ và ý định mua hàng trực tuyến tại Thành phố Nha Trang [Factors affecting attitudes and intention to buy online in Nha Trang City]. Tạp chí Khoa học - Công nghệ Thủy sản, 2, 155-161.

Nunnally, J. C. (1978). Psychometric theory (2nd ed.). New York, NY: McGraw-Hill.

Nunnally, J. C., \& Bernstein, I. H. (1994). Psychometric theory (3rd ed.). New York, NY: McGraw-Hill.

Okazaki, S. (2005). Mobile advertising adoption by multinationals: Senior executive initial responses. Internet Research, 15(2), 160-180.

Pham, H. T. L., \& Tran, M. N. P. (2014). Các nhân tố ảnh hưởng đến thái độ của người tiêu dùng trẻ đối với quảng cáo SMS [Factors influencing young consumers' attitudes toward SMS advertising]. Tạp chí Phát triển Kinh tế, 286, 89-108. 
Pham, T. Q., \& Nguyen, H. N. H. (2017). Các yếu tố ảnh hưởng đến sự thôi thúc mua hàng ngẫu hứng trực tuyến của người tiêu dùng TP.HCM [Factors affecting the urge to buy online impromptu of Ho Chi Minh City consumers]. Tạp chi Khoa hoc Đại hoc Mở Thành phố Hồ Chí Minh, 55(4), 38-50.

Qureshi, I., Fang, Y., Ramsey, E., McCole, P., Ibbotson, P., \& Compeau, D. (2009). Understanding online customer repurchasing intention and the mediating role of trust-An empirical investigation in two developed countries. European Journal of Information Systems, 18(3), 205-222.

Reichheld, F. F., \& Sasser, J. (1996). Zero defections: Quality come to services. Harvard Business Review, 68(5), 105-111.

Sánchez-Fernández, R., \& Iniesta-Bonillo, M. Á. (2007). The concept of perceived value: A systematic review of the research. Marketing Theory, 7(4), 427-451.

Selnes, F. (1993). An examination of the effect of product performance on brand reputation, satisfaction and loyalty. European Journal of Marketing, 27(9), 19-35.

Shavitt, S., Lowrey, P., \& Haefner, J. (1998). Public attitudes toward advertising: More favorable than you might think. Journal of Advertising Research, 38(4), 7-22.

Sheppard, B. H., Hartwick, J., \& Warshaw, P. R. (1988). The theory of reasoned action: A meta-analysis of past research with recommendations for modifications and future research. Journal of Consumer Research, 15(3), 325-343.

Shin, J. I., Chung, K. H., Oh, J. S., \& Lee, C. W. (2013). The effect of site quality on repurchase intention in Internet shopping through mediating variables: The case of university students in South Korea. International Journal of Information Management, 33(3), 453463.

Sinkovics, R. R., Pezderka, N., \& Haghirian, P. (2012). Determinants of consumer perceptions toward mobile advertising - A comparison between Japan and Austria. Journal of Interactive Marketing, 26(1), 21-32.

Steiger, J. H. (1990). Structural model evaluation and modification: An interval estimation approach. Multivariate Behavioral Research, 25(2), 173-180.

Tsai, H. T., \& Huang, H. C. (2007). Determinants of e-repurchase intentions: An integrative model of quadruple retention drivers. Information \& Management, 44(3), 231-239.

Tsang, M. M., Ho, S. C., \& Liang, T. P. (2004). Consumer attitudes toward mobile advertising: An empirical study. International Journal of Electronic Commerce, 8(3), 65-78.

Tu, Y. T. H. (2015). Nghiên cứu các nhân tố tác động đến ý định mua sắm trực tuyến (online shopping) của người tiêu dung [Research the factors affecting the intention of online shopping (online shopping) of consumers]. Tạp chí KH - GD Trưòng Đại học Đông Á, 29-39.

Veloutsou, C., \& Moutinho, L. (2009). Brand relationships through brand reputation and brand tribalism. Journal of Business Research, 62(3), 314-322. 
Wu, L. Y., Chen, K. Y., Chen, P. Y., \& Cheng, S. L. (2014). Perceived value, transaction cost, and repurchase-intention in online shopping: A relational exchange perspective. Journal of Business Research, 67(1), 2768-2776.

Yoon, E., Guffey, H. J., \& Kijewski, V. (1993). The effects of information and company reputation on intentions to buy a business service. Journal of Business Research, 27(3), 215-228.

Zeithaml, V. A. (1988). Consumer perceptions of price, quality, and value: A means-end model and synthesis of evidence. Journal of Marketing, 52(3), 2-22.

Zeng, F., Huang, L., \& Dou, W. (2009). Social factors in user perceptions and responses to advertising in online social networking communities. Journal of Interactive Advertising, 10(1), 1-13.

Zhang, Y., Fang, Y., Wei, K. K., Ramsey, E., McCole, P., \& Chen, H. (2011). Repurchase intention in B2C e-commerce - A relationship quality perspective. Information \& Management, 48(6), 192-200. 